\title{
PROBABILISTIC PROOFS OF EULER IDENTITIES
}

\author{
LARS HOLST, ${ }^{*}$ Royal Institute of Technology
}

\begin{abstract}
Formulae for $\zeta(2 n)$ and $L_{\chi_{4}}(2 n+1)$ involving Euler and tangent numbers are derived using the hyperbolic secant probability distribution and its moment generating function. In particular, the Basel problem, where $\zeta(2)=\pi^{2} / 6$, is considered. Euler's infinite product for the sine is also proved using the distribution of sums of independent hyperbolic secant random variables and a local limit theorem.
\end{abstract}

Keywords: Basel problem; hyperbolic secant distribution; Euler number; tangent number; Euler's sine product

2010 Mathematics Subject Classification: Primary 33B10

Secondary 01A50

\section{Introduction}

Results in mathematical analysis can sometimes be derived and enlightened using probability theory. As an illustration of this 'unity of mathematics', we will see how properties of the socalled hyperbolic secant distribution can be used to obtain Euler's exact sum for the Basel problem from 1735, i.e. the beautiful identity

$$
1+\frac{1}{2^{2}}+\frac{1}{3^{2}}+\cdots=\frac{\pi^{2}}{6}
$$

and more generally to obtain formulae for

$$
\zeta(2 n)=\sum_{k=1}^{\infty} \frac{1}{k^{2 n}}, \quad n=1,2,3, \ldots,
$$

and

$$
L_{\chi_{4}}(2 n+1)=\sum_{k=0}^{\infty} \frac{(-1)^{k}}{(2 k+1)^{2 n+1}}, \quad n=0,1,2, \ldots
$$

The same formulae are derived in [2] using products of Cauchy random variables. We also prove Euler's infinite product formula for the sine using the hyperbolic secant distribution.

\section{The hyperbolic secant probability distribution}

A random variable $X$ with the symmetric probability density

$$
f_{1}(x)=\frac{1}{\pi \cosh x}=\frac{2}{\pi\left(\mathrm{e}^{x}+\mathrm{e}^{-x}\right)}, \quad-\infty<x<\infty,
$$

Received 4 September 2012; revision received 13 February 2013.

* Postal address: Department of Mathematics, Royal Institute of Technology, SE-10044 Stockholm, Sweden. Email address: lholst@math.kth.se 
and the distribution function

$$
\mathbb{P}(X \leq x)=\int_{-\infty}^{x} \frac{2}{\pi\left(\mathrm{e}^{y}+\mathrm{e}^{-y}\right)} \mathrm{d} y=\frac{2}{\pi} \arctan \left(\mathrm{e}^{x}\right),
$$

has a hyperbolic secant (HS) distribution.

Note that, if $Y$ is $C(0,1)$, that is, standard Cauchy distributed, then

$$
\mathbb{P}(\ln |Y| \leq x)=\int_{-\mathrm{e}^{x}}^{\mathrm{e}^{x}} \frac{1}{\pi\left(1+y^{2}\right)} \mathrm{d} y=\frac{2}{\pi} \arctan \left(\mathrm{e}^{x}\right) .
$$

Thus, $\ln |Y|$ is HS distributed.

Theorem 1. Let $X_{1}$ and $X_{2}$ be independent random variables with the HS distribution. Then the probability density of $X_{1}+X_{2}$ is

$$
f_{2}(x)=\frac{4 x}{\pi^{2}\left(\mathrm{e}^{x}-\mathrm{e}^{-x}\right)}, \quad-\infty<x<\infty .
$$

Proof. By convolution, the density of $X_{1}+X_{2}, f_{2}(x)$, is given by

$$
\begin{aligned}
\int_{-\infty}^{\infty} & \frac{2}{\pi\left(\mathrm{e}^{y}+\mathrm{e}^{-y}\right)} \frac{2}{\pi\left(\mathrm{e}^{x-y}+\mathrm{e}^{-x+y}\right)} \mathrm{d} y \\
& =\frac{4}{\pi^{2}} \int_{0}^{\infty} \frac{u \mathrm{e}^{-x}}{\left(1+u^{2}\right)\left(1+u^{2} \mathrm{e}^{-2 x}\right)} \mathrm{d} u \\
& =\frac{4}{\pi^{2}\left(\mathrm{e}^{x}-\mathrm{e}^{-x}\right)} \int_{0}^{\infty}\left(\frac{u}{1+u^{2}}-\frac{u \mathrm{e}^{-2 x}}{1+u^{2} \mathrm{e}^{-2 x}}\right) \mathrm{d} u \\
& =\frac{4 x}{\pi^{2}\left(\mathrm{e}^{x}-\mathrm{e}^{-x}\right)},
\end{aligned}
$$

proving the assertion.

Theorem 2. Let the random variable $X$ have an HS distribution. Then its moment generating function is

$$
\mathbb{E}\left(\mathrm{e}^{t X}\right)=\frac{1}{\cos (\pi t / 2)}, \quad-1<t<1,
$$

and its characteristic function is

$$
\mathbb{E}\left(\mathrm{e}^{\mathrm{i} t X}\right)=\frac{1}{\cosh (\pi t / 2)}=\frac{2}{\mathrm{e}^{\pi t / 2}+\mathrm{e}^{-\pi t / 2}}, \quad-\infty<t<\infty .
$$

Proof. For $-1<t<1$, we have

$$
\mathbb{E}\left(\mathrm{e}^{t X}\right)=\int_{-\infty}^{\infty} \mathrm{e}^{t x} \frac{2}{\pi\left(\mathrm{e}^{x}+\mathrm{e}^{-x}\right)} \mathrm{d} x=\frac{2}{\pi} \int_{0}^{\infty} \frac{y^{t}}{1+y^{2}} \mathrm{~d} y=\frac{1}{\cos (\pi t / 2)},
$$

using a known integral. Replacing $t$ with it proves the second assertion.

Recall the series expansion of the secant function

$$
\frac{1}{\cos t}=\sum_{n=0}^{\infty} E_{2 n} \frac{t^{2 n}}{(2 n) !}, \quad-\frac{\pi}{2}<t<\frac{\pi}{2},
$$


where $E_{0}=1, E_{2}=1, E_{4}=5, E_{6}=61$, etc. are the Euler numbers, and that of the tangent function

$$
\frac{1}{\cos ^{2} t}=\frac{\mathrm{d}}{\mathrm{d} t} \tan t=\frac{\mathrm{d}}{\mathrm{d} t} \sum_{n=0}^{\infty} T_{2 n+1} \frac{t^{2 n+1}}{(2 n+1) !}=\sum_{n=0}^{\infty} T_{2 n+1} \frac{t^{2 n}}{(2 n) !}, \quad-\frac{\pi}{2}<t<\frac{\pi}{2},
$$

where $T_{1}=1, T_{3}=2, T_{5}=16, T_{7}=272$, etc. are the tangent numbers.

For $X_{1}$ and $X_{2}$ independent HS random variables, we have

$$
\mathbb{E}\left(\mathrm{e}^{t\left(X_{1}+X_{2}\right)}\right)=\mathbb{E}\left(\mathrm{e}^{t X_{1}}\right) \mathbb{E}\left(\mathrm{e}^{t X_{2}}\right)=\frac{1}{\cos ^{2}(\pi t / 2)}, \quad-1<t<1 .
$$

From the above properties, the following formulae for the moments follow.

Theorem 3. Let $X_{1}$ and $X_{2}$ be independent HS random variables. Then

$$
\mathbb{E}\left(X_{1}^{2 n}\right)=\mathbb{E}\left(X_{2}^{2 n}\right)=\mathbb{E}_{2 n}\left(\frac{\pi}{2}\right)^{2 n}, \quad \mathbb{E}\left(\left(X_{1}+X_{2}\right)^{2 n}\right)=T_{2 n+1}\left(\frac{\pi}{2}\right)^{2 n},
$$

all odd moments are 0 , and $V\left(X_{1}\right)=V\left(X_{2}\right)=(\pi / 2)^{2}$.

\section{The Basel problem and related identities}

Euler's remarkable exact sum from 1735 for the Basel problem, see, e.g. [3, p. 107], [5, pp. 45-46], [10, p. 38], and [14], has been proved in many different ways; see [4] with 14 ways and [13] with 6 ways for evaluating $\zeta(2)$, and the recent notes [9], [11], [15], and [16]. We give another proof using Theorem 1.

Theorem 4. (The Basel problem.) It holds that

$$
\zeta(2)=1+\frac{1}{2^{2}}+\frac{1}{3^{2}}+\cdots=\frac{\pi^{2}}{6} .
$$

Proof. By symmetry we have

$$
\begin{aligned}
1 & =\int_{-\infty}^{\infty} f_{2}(x) \mathrm{d} x \\
& =\int_{-\infty}^{\infty} \frac{4 x}{\pi^{2}\left(\mathrm{e}^{x}-\mathrm{e}^{-x}\right)} \mathrm{d} x \\
& =\frac{8}{\pi^{2}} \int_{0}^{\infty} \frac{x \mathrm{e}^{-x}}{1-\mathrm{e}^{-2 x}} \mathrm{~d} x \\
& =\frac{8}{\pi^{2}} \int_{0}^{\infty} x \mathrm{e}^{-x} \sum_{k=0}^{\infty} \mathrm{e}^{-2 k x} \mathrm{~d} x \\
& =\frac{8}{\pi^{2}} \sum_{k=0}^{\infty} \int_{0}^{\infty} x \mathrm{e}^{-(2 k+1) x} \mathrm{~d} x \\
& =\frac{8}{\pi^{2}} \sum_{k=0}^{\infty} \frac{1}{(2 k+1)^{2}} .
\end{aligned}
$$


Euler's identity follows from

$$
\zeta(2)=\sum_{k=0}^{\infty} \frac{1}{(2 k+1)^{2}}+\sum_{k=1}^{\infty} \frac{1}{(2 k)^{2}}=\frac{\pi^{2}}{8}+\frac{1}{4} \zeta(2) .
$$

The following theorem, which partly goes back to Euler, is proved in a similar way. A slightly different proof is given in [2]; cf. [16].

Theorem 5. For $n=1,2,3, \ldots$,

$$
\left(1-\frac{1}{2^{2 n}}\right) \zeta(2 n)=\frac{1}{2}\left(\frac{\pi}{2}\right)^{2 n} \frac{T_{2 n-1}}{(2 n-1) !}
$$

and, for $n=0,1,2, \ldots$,

$$
L_{\chi_{4}}(2 n+1)=\sum_{k=0}^{\infty} \frac{(-1)^{k}}{(2 k+1)^{2 n+1}}=\frac{1}{2}\left(\frac{\pi}{2}\right)^{2 n+1} \frac{E_{2 n}}{(2 n) !} .
$$

Proof. From Theorem 1 and Theorem 3, we obtain

$$
\begin{aligned}
T_{2 n-1}\left(\frac{\pi}{2}\right)^{2 n-2} & =\mathbb{E}\left(\left(X_{1}+X_{2}\right)^{2 n-2}\right) \\
& =\int_{-\infty}^{\infty} x^{2 n-2} f_{2}(x) \mathrm{d} x \\
& =\frac{8}{\pi^{2}} \sum_{k=0}^{\infty} \int_{0}^{\infty} x^{2 n-1} \mathrm{e}^{-(2 k+1) x} \mathrm{~d} x \\
& =\frac{8}{\pi^{2}} \sum_{k=0}^{\infty} \frac{(2 n-1) !}{(2 k+1)^{2 n}} .
\end{aligned}
$$

The first assertion follows as in the previous theorem.

In a similar way the second assertion follows from

$$
E_{2 n}\left(\frac{\pi}{2}\right)^{2 n}=\int_{-\infty}^{\infty} x^{2 n} f_{1}(x) \mathrm{d} x=\frac{4}{\pi} \sum_{k=0}^{\infty}(-1)^{k} \int_{0}^{\infty} x^{2 n} \mathrm{e}^{-(2 k+1) x} \mathrm{~d} x
$$

\section{Sums of independent HS random variables}

Theorem 6. Let $X_{1}, X_{2}, \ldots, X_{n}$ be independent $H S$ random variables, and let $f_{n}(x)$ be the probability density of $X_{1}+\cdots+X_{n}$. Then, for $k=1,2, \ldots$,

$$
\begin{gathered}
f_{2 k+1}(x)=\frac{(2 k-1) ! !}{(2 k) ! !} \prod_{j=1}^{k}\left(1+\left(\frac{2 x}{(2 j-1) \pi}\right)^{2}\right) \frac{2}{\pi\left(\mathrm{e}^{x}+\mathrm{e}^{-x}\right)}, \\
f_{2 k+2}(x)=\frac{(2 k) ! !}{(2 k+1) ! !} \prod_{j=1}^{k}\left(1+\left(\frac{x}{j \pi}\right)^{2}\right) \frac{4 x}{\pi^{2}\left(\mathrm{e}^{x}-\mathrm{e}^{-x}\right)},
\end{gathered}
$$

and, uniformly in $y$,

$$
\frac{\pi \sqrt{n}}{2} f_{n}\left(\frac{\pi \sqrt{n}}{2} y\right) \rightarrow \frac{1}{\sqrt{2 \pi}} \mathrm{e}^{-y^{2} / 2} \text { as } n \rightarrow \infty .
$$


Proof. Using Theorem 2, we have, for $-1<t<1$,

$$
\begin{aligned}
\frac{\mathrm{d}^{2}}{\mathrm{~d} t^{2}} \mathbb{E}\left(\mathrm{e}^{t\left(X_{1}+\cdots+X_{n}\right)}\right) & =\frac{\mathrm{d}^{2}}{\mathrm{~d} t^{2}} \int_{-\infty}^{\infty} \mathrm{e}^{t x} f_{n}(x) \mathrm{d} x \\
& =\int_{-\infty}^{\infty} \mathrm{e}^{t x} x^{2} f_{n}(x) \mathrm{d} x \\
& =\frac{\mathrm{d}^{2}}{\mathrm{~d} t^{2}} \frac{1}{\cos ^{n}(\pi t / 2)} \\
& =\frac{n(n+1) \pi^{2}}{4 \cos ^{n+2}(\pi t / 2)}-\frac{n^{2} \pi^{2}}{4 \cos ^{n}(\pi t / 2)} \\
& =\frac{n(n+1) \pi^{2}}{4} \int_{-\infty}^{\infty} \mathrm{e}^{t x} f_{n+2}(x) \mathrm{d} x-\frac{n^{2} \pi^{2}}{4} \int_{-\infty}^{\infty} \mathrm{e}^{t x} f_{n}(x) \mathrm{d} x .
\end{aligned}
$$

Thus,

$$
\int_{-\infty}^{\infty} \mathrm{e}^{t x} f_{n+2}(x) \mathrm{d} x=\int_{-\infty}^{\infty} \mathrm{e}^{t x} \frac{n}{n+1}\left(1+\left(\frac{2 x}{n \pi}\right)^{2}\right) f_{n}(x) \mathrm{d} x, \quad-1<t<1,
$$

which implies the recurrence

$$
f_{n+2}(x)=\frac{n}{n+1}\left(1+\left(\frac{2 x}{n \pi}\right)^{2}\right) f_{n}(x) .
$$

This is satisfied by

$$
f_{2 k+1}(x)=\frac{(2 k-1) ! !}{(2 k) ! !} \prod_{j=1}^{k}\left(1+\left(\frac{2 x}{(2 j-1) \pi}\right)^{2}\right) f_{1}(x)
$$

and

$$
f_{2 k+2}(x)=\frac{(2 k) ! !}{(2 k+1) ! !} \prod_{j=1}^{k}\left(1+\left(\frac{x}{j \pi}\right)^{2}\right) f_{2}(x)
$$

proving the first two assertions.

By the central limit theorem, $\left(X_{1}+\cdots+X_{n}\right) /(\pi \sqrt{n} / 2)$ converges in distribution to a standard normal distribution as $n \rightarrow \infty$. From Theorem 2 we see that the characteristic function of $X$ is integrable, which implies that the probability densities converge to the standard normal probability density

$$
\frac{\pi \sqrt{n}}{2} f_{n}\left(\frac{y \pi \sqrt{n}}{2}\right) \rightarrow \frac{1}{\sqrt{2 \pi}} \mathrm{e}^{-y^{2} / 2} \text { as } n \rightarrow \infty,
$$

uniformly in $y$; see [6, Theorem 2, p. 489].

By other methods, the formulae for $f_{2 k+1}(x)$ and $f_{2 k+2}(x)$ are derived in, e.g. [1] and [8].

Remark. Recall that $\ln |Y|$ has an HS distribution for $Y$ standard Cauchy distributed. Thus, products and ratios of independent $C(0,1)$ random variables can be studied using sums of independent HS random variables; cf. [2, Proposition 2], [8], and [16]. 


\section{Euler's product formula for the sine}

Using the results for sums of HS random variables, we now prove Euler's infinite product formula for the sine from 1735. For other proofs, see, e.g. [7], the references therein, and the recent note [12].

Theorem 7. For all complex $z$,

$$
\sin z=z \prod_{j=1}^{\infty}\left(1-\frac{z^{2}}{j^{2} \pi^{2}}\right)
$$

Proof. Using Theorem 6, we obtain

$$
f_{2 k+2}(x)=\frac{2^{2 k+1}(k !)^{2}}{(2 k) !(2 k+1) \pi^{2}} \frac{2}{\mathrm{e}^{x}-\mathrm{e}^{-x}} x \prod_{j=1}^{k}\left(1+\frac{x^{2}}{j^{2} \pi^{2}}\right) .
$$

By Stirling's formula,

$$
\frac{2^{2 k+1}(k !)^{2}}{(2 k) ! \sqrt{2 k+1}} \rightarrow \sqrt{2 \pi} \quad \text { as } k \rightarrow \infty .
$$

The uniform convergence of the densities implies that, for every fixed $x$,

$$
\frac{\pi \sqrt{2 k+1}}{2} f_{2 k+2}(x) \rightarrow \frac{1}{\sqrt{2 \pi}} \quad \text { as } k \rightarrow \infty .
$$

Thus,

$$
\frac{(2 k) !(2 k+1) \pi^{2}}{2^{2 k+1}(k !)^{2}} f_{2 k+2}(x) \rightarrow 1 \quad \text { as } k \rightarrow \infty .
$$

Combining the above limits, we obtain

$$
\sinh x=\frac{\mathrm{e}^{x}-\mathrm{e}^{-x}}{2}=x \prod_{j=1}^{\infty}\left(1+\frac{x^{2}}{j^{2} \pi^{2}}\right) .
$$

As this holds for all real $x$, it holds by analytical continuation for all complex $x$. With $x=\mathrm{i} z$, Euler's product formula follows.

\section{References}

[1] BATEN, W. D. (1934). The probability law for the sum of $n$ independent variables, each subject to the law $(1 /(2 h)) \operatorname{sech}(\pi x /(2 h))$. Bull. Amer. Math. Soc. 40, 284-290.

[2] Bourgade, P., Fujita, T. And Yor, M. (2007). Euler's formulae for $\zeta(2 n)$ and products of Cauchy variables. Electron. Commun. Prob. 12, 73-80.

[3] Bradley, R. E., D’Antonio, L. A. And Sandifer, C. E. (eds) (2007). Euler at 300. An Appreciation. Mathematical Association of America, Washington, DC.

[4] Chapman, R. (2003). Evaluating $\zeta(2)$. Preprint. Available at http://www.uam.es/personal_pdi/ciencias/cillerue/ Curso/zeta2.pdf

[5] Dunham, W. (1999). Euler: The Master of Us All. Mathematical Association of America, Washington, DC.

[6] Feller, W. (1966). An Introduction to Probability Theory and Its Applications, Vol. 2. John Wiley, New York.

[7] Gordon, L. (1994). A stochastic approach to the gamma function. Amer. Math. Monthly 101, 858-865.

[8] Harkness, W. L. and Harkness, M. L. (1968). Generalized hyperbolic secant distributions. J. Amer. Statist. Assoc. 63, 329-337.

[9] Harper, J. D. (2003). Another simple proof of $1+1 / 2^{2}+1 / 3^{2}+\cdots=\pi^{2} / 6$. Amer. Math. Monthly 110, 540-541. 
[10] Havil, J. (2003). Gamma: Exploring Euler's Constant. Princeton University Press.

[11] Hofbauer, J. (2002). A simple proof of $1+1 / 2^{2}+1 / 3^{2}+\cdots=\pi^{2} / 6$ and related identities. Amer. Math. Monthly 109, 196-200.

[12] Holst, L. (2012). A proof of Euler's infinite product for the sine. Amer. Math. Monthly 119, 518-521.

[13] Kalman, D. (1993). Six ways to sum a series. College Math. J. 24, 402-421.

[14] Kalman, D. And McKinzie, M. (2012). Another way to sum a series: generating functions, Euler, and the dilog function. Amer. Math. Monthly 119, 42-51.

[15] Marshall, T. (2010). A short proof of $\zeta(2)=\pi^{2} / 6$. Amer. Math. Monthly 117, 352-353.

[16] PACE, L. (2011). Probabilistically proving that $\zeta(2)=\pi^{2} / 6$. Amer. Math. Monthly 118, 641-643. 